\title{
SISTEMA DE AUTOMATIZACIÓN DE UN CALDERO DE 30 BHP PARA EL MEJORAMIENTO DE LA EFICIENCIA ENERGÉTICA UTILIZANDO VARIABLES TERMODINÁMICAS
}

\author{
AUTOMATION SYSTEM OF A 30 HP BOILER TO IMPROVE \\ ENERGY EFFICIENCY USING THERMODYNAMIC VARIABLES
}

\author{
Ubaldo Apaza $^{1}$, Aldo Delgado ${ }^{2}$, Iván Garcilazo ${ }^{3}$, Igor Obregón ${ }^{4}$ \\ RESUMEN
}

Se realizó la implementación de un sistema de control automático en el caldero pirotubular del Laboratorio de Operaciones Unitarias de la Facultad de Ingeniería Química y Textil para mejorar el aprovechamiento del vapor generado por este. Los puntos implementados fueron, un sistema de purga automático empleando un lenguaje de programación ladder establecido en un relé programable. Este sistema permite la purga automática, mediante una válvula neumática; el segundo punto fue la implementación de un sistema de monitoreo, supervisión y registro de datos de la temperatura de gases de combustión y como último punto, la implementación de una analizador portátil de gases de combustión y el aislamiento total de conductos de vapor para minimizar las pérdidas de energía. Los mejores resultados de esta investigación podrán ser aplicados, bajo el mismo procedimiento, a nivel industrial en industrias que usen las calderas como su corazón para sus diversos procesos con el fin de obtener mejoras en sus procesos y en sus costos de operación.

Palabras clave.-_Ladder, Purga, Caldera, Relé programable, Sólidos disueltos.

\begin{abstract}
The implementation of an automatic control system in the fire-tube boiler of the Unit Operations Laboratory of the FIQT-UNI was performed to improve the utilization of the steam generated by it. The first point implemented had been an automatic purge using a ladder programming language established in a relay. This system allows the automatic purge by using a pneumatic valve; the second point was the implementation of a monitoring system, supervision and data logging of the temperature flue gas and as a last point, the implementation of a portable flue gas analyzer and the total steam duct insulation in order to minimize energy losses.The best results of this research could be applied, through the same process, on an industrial level in industries which use boilers as their heart for their various processes in order to obtain improvements in their processes and in their operation costs.
\end{abstract}

Key words.- Ladder, Purge, Boiler, Programmable relay, Dissolved solids.

\section{INTRODUCCIÓN}

En estos días la mayoría de nuestras actividades están basadas en el consumo de combustibles fósiles, los cuales son fuentes de energía no renovables es por ello que la prioridad es sacar el máximo beneficio en cuanto a la energía producida a partir de ellos [1].

\footnotetext{
${ }^{1}$ Ing. Investigador y docente de la Facultad de Ingeniería Química y Textil de la Universidad Nacional de Ingeniería, ${ }^{2}$ Mag. Investigador, Docente de la Facultad de Ingeniería Química y Textil de la Universidad Nacional de Ingeniería, ${ }^{3}$ Bach. Investigador de la Facultad de Ingeniería Química y Textil de la Universidad Nacional de Ingeniería, ${ }^{4}$ Bach. Investigador de la Facultad de Ingeniería Química y Textil de la Universidad Nacional de Ingeniería.
} 
Como resultado de este consumo se producen importantes elementos que aceleran el calentamiento global tales como el $\mathrm{CO}_{2}, \mathrm{CO}, \mathrm{SO}_{\mathrm{x}}$ y $\mathrm{NO}_{\mathrm{x}}$, la producción de los 2 primeros es inevitable, por ende se debe de asegurar que genere lo mínimo posible, de los últimos se debe asegurar la calidad del combustible antes de usarse $\left[\begin{array}{lll}2 & \text { y } 3] \text {. }\end{array}\right.$

Por lo anterior, las calderas son grandes consumidores de combustible fósil y también generadores de diversos gases de combustión [2]. $\mathrm{Su}$ fin es aprovechar la energía térmica generada en la combustión para trasformar el agua líquida en vapor para satisfacer las diferentes demandas de la industria [4]. Se puede hacer uso de modernas técnicas para mejorar el aprovechamiento de dicha energía. Para el caso de una caldera de vapor se requiere un simulador para observar su desempeño operativo y mejorar su eficiencia como equipo para generar expectativas técnicas favorables, que llevándolo a un plano industrial, se refiere a dos puntos, ahorro económico y menor contaminación. [5].

El control de los lodos de fondos al interior de una caldera es muy importante debido a que un acumulamiento excesivo generaría una capa aislante entre el exterior del hogar y el agua, disminuyendo la conductividad calorífica de la pared exterior del hogar y por ende existiría la necesidad de aumentar la temperatura para generar la misma transferencia de calor entre la energía de la combustión del combustible y el agua de caldera. Esta necesidad de aumento de temperatura tiene como consecuencia inmediata el consumo de más combustible por parte de la caldera y como impacto ambiental a este, la generación de más gases de combustión en el ambiente aumentando su concentración como consecuencia de esto se tendría un foco adicional que contribuye a acelerar el calentamiento global.

La siguiente investigación nos muestra un método para determinar el intervalo de tiempo de purgado de una caldera pirotubular y su accionar mediante un sistema automatizado [6].

\section{MATERIALES Y METODOLGÍA}

\section{Materiales}

El programa ladder (programa de automatización) fue realizado en un relé programable compacto
Zelio Logic [7], del cual se conectó la salida al esclavo, una válvula ON-OFF (válvula de purgado), el cual controla con la purga los sólidos disueltos totales dentro de la caldera.

Se instaló un RTD (PT100) en el ducto de gases de combustión para la medición de la temperatura, esta señal analógica fue llevada al adquisidor de datos ICP DAS [8] para su registro y transformación de señal, teniendo una salida Ethernet, para ser enviando a la pC, el cual se le instalado un Scada de supervisión, que permite monitorear las variables de la caldera, que en este caso es la purga de fondos y la temperatura de chimenea

\section{Mantenimiento total de la caldera}

En esta primera etapa se analizó las condiciones del exterior de la caldera las cuales no era muy convenientes, debido a que su superficie durante el funcionamiento se mantenía a una alta temperatura, así como las tuberías por donde se conducía el vapor generado, esto era un pésimo sistema debido a que se perdía el calor por medio de las paredes de la caldera y conductos de la caldera, debido a que toda la energía que llevaba el vapor se iba perdiendo por estos, es por ello que lo primero que se realizó fue darle un mejor aislamiento a la superficie de la caldera que se cubrió con empaquetaduras de acero al carbono a su alrededor para darle una buena presentación, entre el acero al carbono y la superficie de la caldera se rellenó con fibra de vidrio para asilar con mayor rigurosidad.

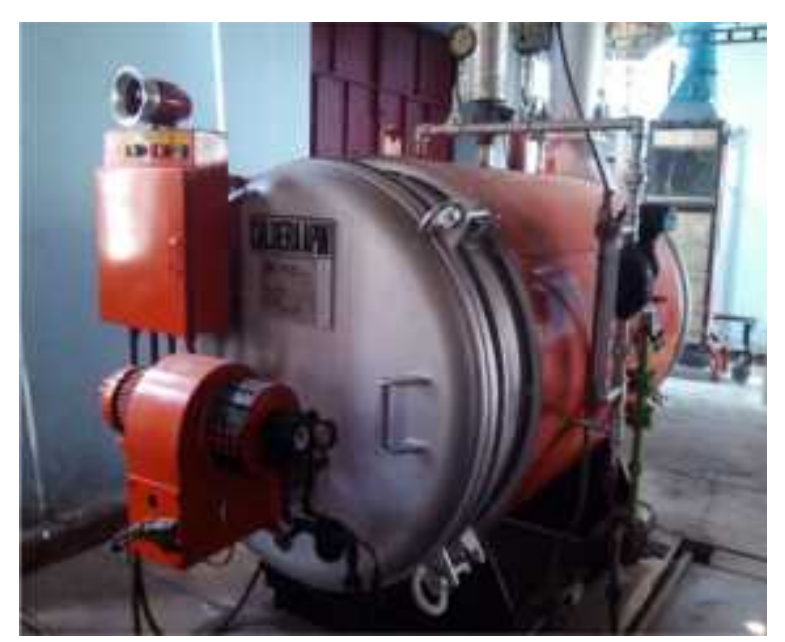

Fig. 1 Aislantes colocados sobre la superficie de la caldera y el ducto de vapor. 


\section{Implementación del nuevo sistema de purga}

El sistema manual de purga fue sustituido por un nuevo sistema neumático de purga que es accionado por aire comprimido. La válvula manual puede ser regulada para que al abrirse la válvula neumática $(\mathrm{ON}-\mathrm{OFF})$, el agua de fondo no fluya con mucha presión.

\section{Automatización del sistema de purga}

En el relé programable Zelio Logic, que se programó en lenguaje ladder, que permito enviar la señal a la válvula ON-OFF para que se accione de acuerdo a un tiempo previamente programado y un determinado número de intervalos para evitar la pérdida de energía por el agua purgada. Estos tiempos son mostrados en la discusión de resultados.

\section{Sensor de temperatura - RTD}

La señal del detector de temperatura resistivo RTD - PT100 que está colocado en el ducto de la chimenea de la caldera, es enviada a un Adquisidor de datos (DAQ), para su posterior registro y monitoreo en un SCADA.
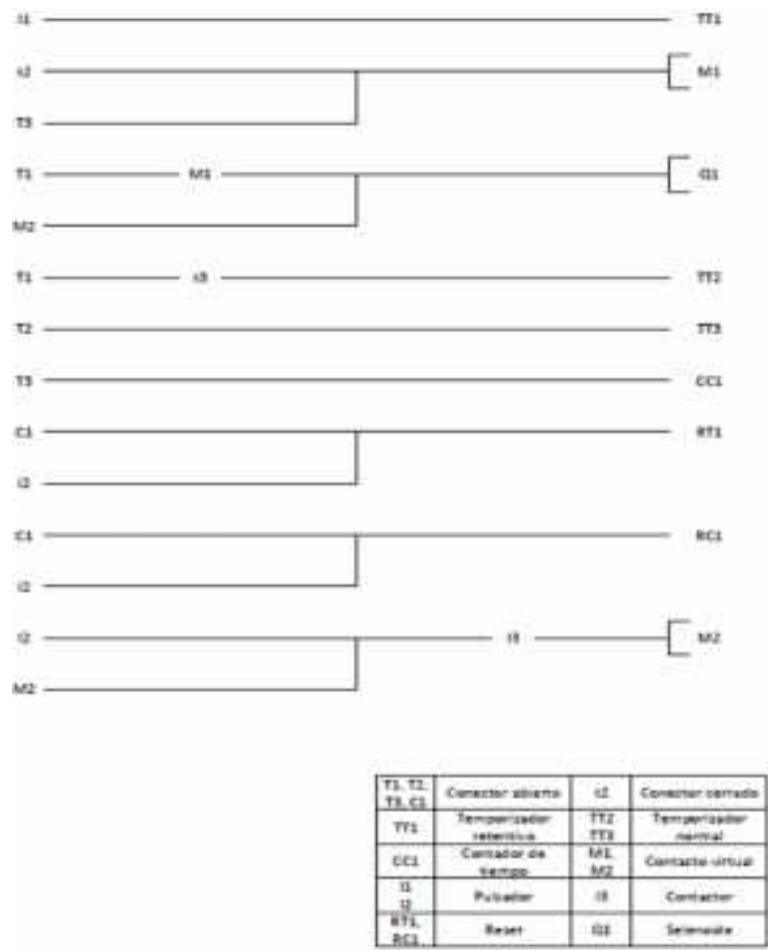

Fig. 2 Programa ladder para la apertura automática de la válvula de purga.

\section{Monitoreo del proyecto}

El monitoreo continuo del proyecto es realizado mediante el uso del Scada - software Indusoft [9], el cual es una herramienta muy versátil para poder monitorear y supervisar el proceso. En este caso, tenemos dos variables para monitorear, la purga y la temperatura de la chimenea.

El Scada nos da una lectura a tiempo real de la temperatura de la chimenea en cada instante de tiempo, así como el estado de la válvula de purga la cual es una variable booleana puesto que sólo tiene dos valores (abierto o cerrado).

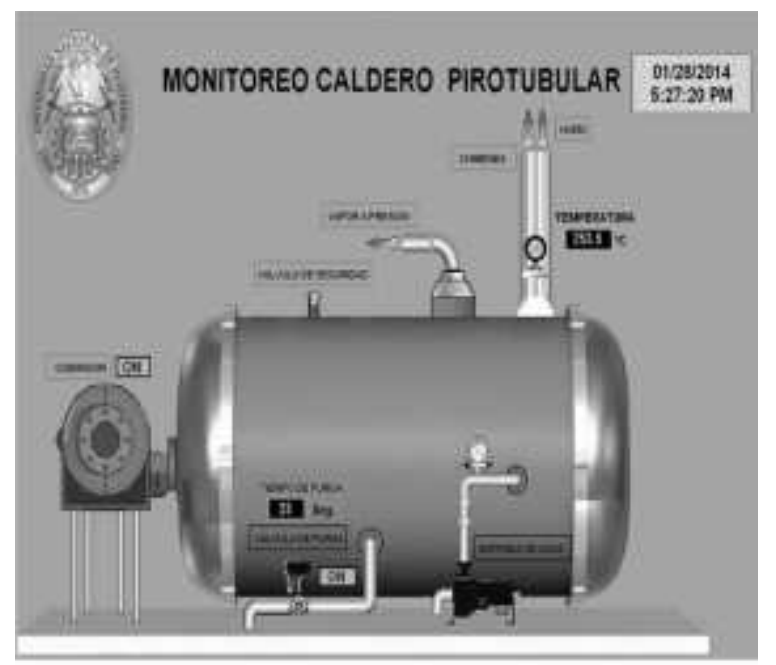

Fig. 3 Monitoreo y supervisión del caldero pirotubular a través del Scada - software Indusoft.

\section{RESULTADO Y DISCUSIÓN}

La Tabla 1 muestra los resultados de las mediciones del agua de purga sin efectuar ingreso de agua a la caldera. Se realizaron tres muestras y en ellas se midió con un conductímetro portátil los valores de conductividad, sólidos totales disueltos, temperatura del agua.

La temperatura se dejó enfriar hasta lograr una temperatura ambiente, para poder comprar los valores obtenidos con los valores de tablas para los límites máximos de sólidos totales disueltos.

También se tomó en consideración la hora de la muestra y el tiempo que demora el purgado. 
Tabla 1. Conductividad y STD de la purga sin ingreso de agua a la caldera.

\begin{tabular}{llll}
\hline \multicolumn{1}{c}{ Muestra } & \multicolumn{1}{c}{$\mathbf{1}$} & \multicolumn{1}{c}{$\mathbf{2}$} & \multicolumn{1}{c}{$\mathbf{3}$} \\
& & & \\
\hline Conductividad $(\mathrm{mS} / \mathrm{cm})$ & 11.2 & 9.91 & 7.79 \\
$\mathrm{STD}(\mathrm{g} / \mathrm{l})$ & 5.91 & 5.31 & 4.15 \\
Temperatura $\left({ }^{\circ} \mathrm{C}\right)$ & 23.0 & 23.0 & 23.0 \\
Hora de muestra & $07: 50$ & $08: 05$ & $08: 20$ \\
Tiempo de purga & 3 & 3 & 3 \\
\hline \multicolumn{1}{c}{ Muestra } & & & \\
\hline & & & \\
& & $\mathbf{5}$ & $\mathbf{6}$ \\
\hline Conductividad $(\mathrm{mS} / \mathrm{cm})$ & 6.69 & 5.85 & 4.51 \\
STD $(\mathrm{g} / \mathrm{l})$ & 3.15 & 2.45 & 1.68 \\
Temperatura $\left({ }^{\circ} \mathrm{C}\right)$ & 23.0 & 23.0 & 23.0 \\
Hora de muestra & $08: 30$ & $09: 05$ & $09: 42$ \\
Tiempo de purga & 3 & 3 & 3 \\
\hline
\end{tabular}

En la Figura 4 proveniente de la Tabla 1, se observa una tendencia decreciente cuando en cuanto al valor de STD (g/l) conforme se va tomando las muestras cuando la caldera funciona sin ingreso de agua.

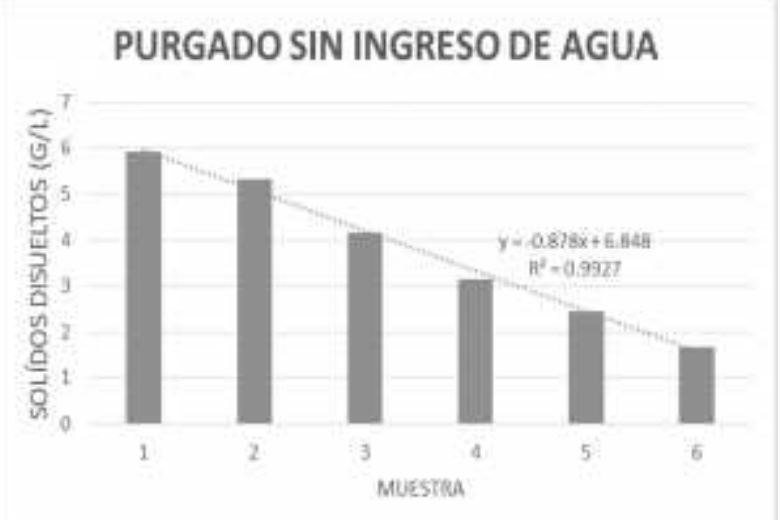

Fig. 4 Decrecimiento en la concentración de sólidos totales disueltos.

La Tabla 2 muestra las mismas variables que la Tabla 1 a diferencia que en esta ocasión se realizó las operaciones con la bomba accionada, la cual permitía el ingreso de agua al interior de la caldera.

Tabla 2. Conductividad y STD de la purga con ingreso de agua a la caldera.

\begin{tabular}{|c|c|c|c|c|}
\hline Muestra & & & & 3 \\
\hline Conductividad (mS/cm) & 7.27 & 7.32 & 7.38 & \\
\hline $\operatorname{STD}(g / l)$ & 3.89 & 3.9 & 3.96 & \\
\hline Tempera $\left({ }^{\circ} \mathrm{C}\right)$ & 23.0 & 23.0 & 23.0 & \\
\hline Hora de muestra & $10: 10$ & 10.18 & 10.48 & \\
\hline Tiempo transcurrido (min) & 0 & 8 & 38 & \\
\hline Muestra & & & & 5 \\
\hline Conductividad (mS/cm) & 7.40 & 7.42 & 7.66 & \\
\hline $\operatorname{STD}(g / l)$ & 3.97 & 3.98 & 4.12 & \\
\hline Tempera $\left({ }^{\circ} \mathrm{C}\right)$ & 23.0 & 23.0 & 23.0 & \\
\hline Hora de muestra & $10: 55$ & 11:04 & $11: 15$ & \\
\hline Tiempo transcurrido (min) & 48 & 58 & 65 & \\
\hline Muestra & & & & 8 \\
\hline Conductividad (mS/cm) & 7.67 & 7.70 & 7.72 & \\
\hline $\operatorname{STD}(g / l)$ & 4.13 & 4.15 & 4.20 & \\
\hline Tempera $\left({ }^{\circ} \mathrm{C}\right)$ & 23.0 & 23.0 & 23.0 & \\
\hline Hora de muestra & $11: 38$ & $11: 44$ & $11: 54$ & \\
\hline Tiempo transcurrido (min) & 88 & 94 & 104 & \\
\hline
\end{tabular}


La Figura 5 muestra la tendencia creciente de la concentración de STD $(\mathrm{g} / \mathrm{l})$ conforme trascurre el tiempo de la toma de muestra.

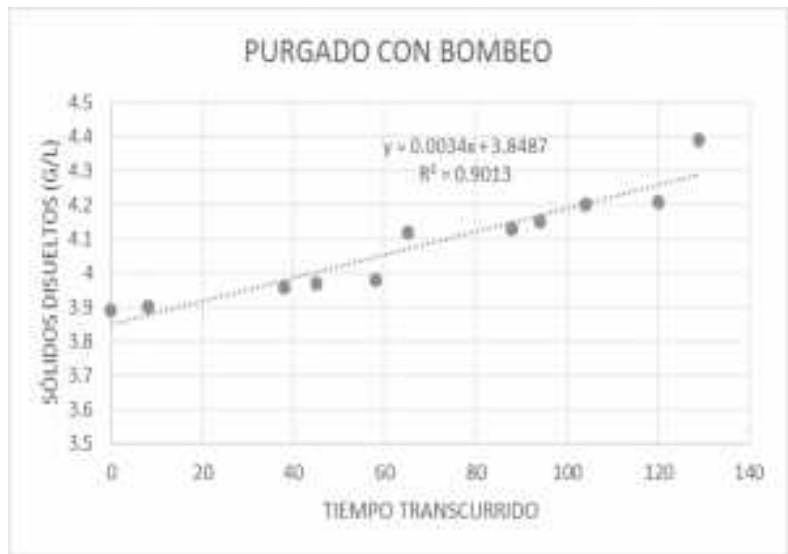

Fig. 5 Crecimiento en la concentración de sólidos totales disueltos.
Se midió previamente que los STD del agua que ingresa a la caldera son de $1356 \mathrm{mg} / \mathrm{l}$, la cual será la cota mínima.

La caldera trabaja a una presión de 150 psi, para esta presión la concentración STD máxima es de $3500 \mathrm{mg} / 1$ [10], la cual será nuestra cota máxima.

La velocidad de aumento de la concentración de STD de la Figura 5 es de 0.0034 g/l.min, mientras que la velocidad de decremento de la concentración de STD de la Figura 4 es de 0.895 $\mathrm{g} / \mathrm{l}$ esto con un intervalo de 3 segundos de purga, que hace una velocidad de $0.2983 \mathrm{~g} / \mathrm{l} . \mathrm{s}$.

La Tabla 3 muestra el tiempo para empezar a realizar la purga en la caldera el cual se estimó en base a las velocidades de variación de STD de la Figura 4 y 5 .

Tabla 3. Cota inferior y cota superior para diferente tiempo de purga.

\begin{tabular}{c|c|c}
\hline $\begin{array}{c}\text { Solidos Totales disueltos }(\mathrm{g} / \mathrm{l}) \\
\text { cota inferior }\end{array}$ & $\begin{array}{c}\text { Solidos Totales disueltos }(\mathrm{g} / \mathrm{l}) \\
\text { Superior }\end{array}$ & $\begin{array}{c}\text { cota } \\
\text { Tiempo para empezar a purgar } \\
\text { (horas) }\end{array}$ \\
\hline 1,356 & 3,5 & 10,5 \\
1,856 & 3,5 & 8,1 \\
2,356 & 3,5 & 5,6 \\
2,856 & 3,5 & 3,2 \\
3,356 & 3,5 & 0,7 \\
\hline
\end{tabular}

El tiempo de apertura de la válvula se obtiene de la relación entre la velocidad de decrecimiento de STD y el límite máximo permisible de la concentración de STD, usando la ecuación (1).

$$
\begin{gathered}
\mathrm{t}_{\text {apertura }}=\frac{\mathrm{LMP}_{\mathrm{STD}}}{\text { velocidad }_{[\mathrm{STD}]}} \\
\mathrm{t}_{\text {apertura }}=\frac{3.5 \frac{\mathrm{g}}{1}}{0.293 \frac{\mathrm{g}}{1 . \mathrm{s}}} \\
\mathrm{t}_{\text {apertura }}=7.2 \mathrm{~s}
\end{gathered}
$$

Por razones de velocidad de abertura total de la válvula ON-OFF se debe asignar un tiempo adicional, el cual será hasta 9 segundos.

Finalmente, se comprobó la conexión del SCADA para la toma de datos tomando la temperatura de la chimenea durante un intervalo de tiempo al realizar las modificaciones en el tiempo de apertura de la válvula de purga y el intervalo de purga. Los datos de la Tabla 4 fueron capturados dentro de un archivo del software Indusoft y luego exportados a un Excel. 
Tabla 4. Temperatura de la chimenea medida con el RTD Pt100 y enviada al SCADA.

\begin{tabular}{|c|c|}
\hline Fecha $_{\text {muestra }}$ & $\mathbf{T}_{\text {chimenea }}\left({ }^{\circ} \mathbf{C}\right)$ \\
\hline 10/07/2014 14:22 & 81.7430 \\
\hline 10/07/2014 14:23 & 101.9385 \\
\hline 10/07/2014 14:24 & 109.2860 \\
\hline 10/07/2014 14:25 & 113.5074 \\
\hline 10/07/2014 14:26 & 104.8653 \\
\hline 10/07/2014 14:27 & 98.1230 \\
\hline 10/07/2014 14:28 & 92.9954 \\
\hline 10/07/2014 14:29 & 93.7196 \\
\hline 10/07/2014 14:30 & 104.9803 \\
\hline 10/07/2014 14:31 & 114.9368 \\
\hline 10/07/2014 14:32 & 121.7210 \\
\hline 10/07/2014 14:33 & 126.2562 \\
\hline 10/07/2014 14:34 & 129.4677 \\
\hline 10/07/2014 14:35 & 131.8902 \\
\hline 10/07/2014 14:36 & 133.8453 \\
\hline 10/07/2014 14:37 & 135.8422 \\
\hline 10/07/2014 14:38 & 138.0797 \\
\hline 10/07/2014 14:39 & 140.3667 \\
\hline 10/07/2014 14:40 & 142.7744 \\
\hline 10/07/2014 14:41 & 145.1960 \\
\hline 10/07/2014 14:42 & 147.5758 \\
\hline 10/07/2014 14:43 & 149.7866 \\
\hline 10/07/2014 14:44 & 151.7481 \\
\hline 10/07/2014 14:45 & 153.5229 \\
\hline 10/07/2014 14:46 & 155.1724 \\
\hline 10/07/2014 14:47 & 156.8250 \\
\hline 10/07/2014 14:48 & 158.3634 \\
\hline 10/07/2014 14:49 & 159.8983 \\
\hline 10/07/2014 14:50 & 161.3737 \\
\hline 10/07/2014 14:51 & 162.7787 \\
\hline 10/07/2014 14:52 & 164.2217 \\
\hline 10/07/2014 14:53 & 165.6704 \\
\hline 10/07/2014 14:54 & 167.0683 \\
\hline 10/07/2014 14:55 & 168.3099 \\
\hline 10/07/2014 14:56 & 169.6925 \\
\hline 10/07/2014 14:57 & 171.0400 \\
\hline 10/07/2014 14:58 & 172.3660 \\
\hline 10/07/2014 14:59 & 173.6047 \\
\hline 10/07/2014 15:00 & 174.9636 \\
\hline 10/07/2014 15:01 & 176.3081 \\
\hline
\end{tabular}

\section{CONCLUSIONES}

Este trabajo determinó el intervalo de purga óptimo para lograr reducir la concentración de los sólidos totales disueltos del agua de fondo de la caldera pirotubular de 30 BHP de la Facultad de Ingeniería Química y Textil y que al mismo tiempo evite una mayor pérdida de energía desaprovechada en el agua que se elimina, por último haciendo uso de esta única variable se evita el consumo de más combustible lo que genera una menor producción de $\mathrm{CO}_{2}$ y otros gases de combustión. Este intervalo óptimo de purgado es de 10,5 horas.

Siguiendo con el lado de ahorro energético, se determinó el tiempo de apertura de la válvula de purga para lograr la reducción de STD del agua de caldera. Esto evita que se eliminé más agua y se pierda la energía que lleva consigo esa agua. Por lo tanto el accionar de la purga es de 9 segundos, con 3 intervalos de 3 segundos cada uno, teniendo un tiempo muerto de 2 segundos.

La velocidad de la concentración de STD de los lodos de fondo de la caldera aumenta al realizar el purgado con ingreso de agua, mientras que dicha velocidad disminuye si el purgado se efectúa sin ingreso de agua a la caldera.

El sistema de purga de caldero evitará darle un mantenimiento más seguido a la caldera. Además, los riesgos a la salud para los operadores que manejen la caldera serán reducidos porque no tendrán contacto al momento de abrirse la válvula de purga.

El monitoreo a distancia del sistema del caldero evitará las paradas innecesarias al caldero, debido a la visualización de la temperatura de los gases de combustión, lo que permitirá realizar una acción preventiva si se observa un comportamiento anómalo, lo cual contribuye a disminuir el tiempo en el cual se solucionas las eventuales fallas.

El SCADA (Indusoft) tiene potencial para la implementación de más puntos de control, con lo cual se reducirá los gastos de una automatización total del sistema, ya que no implica cambios en la lógica de programación.

Los datos descargados por el software Indusoft tienen más confiabilidad que tomados de manera 
visual y manual. Debido a que la información enviada por el relé es almacenada en el software y exportados a un Excel donde se puede realizar el tratamiento deseado

Con el remodelamiento de la caldera en conjunto, se aumentó la fiabilidad y seguridad del sistema, requerimiento muy importante en la generación de vapor.

\section{AGRADECIMIENTOS}

El Grupo de Investigación quiere agradecer al Ministerio de la Producción por las facilidades brindadas para poder tener una visión más amplia sobre el papel fundamental que cumplen las calderas industriales en nuestra industria, de manera más precisa en la industria pesquera. De este modo este trabajo espera ser de vital ayuda en la labor realizada por el Ministerio de la Producción en pos de la eficiencia energética de Calderas Industriales.

\section{REFERENCIAS}

1. Ministerio de Educación, Política Social y Deporte, "Fuentes de Energía para el Futuro", editorial Estilo Estugraf Impresores, S.L., Madrid 2008.

2. Esquerra Pizà, P., "Dispositivos y Sistemas para el ahorro de energía", editorial Marcombo S.A., Barcelona 1988.

3. Dondero Villanueva, L. Z., Texeira Cohelho, S., "La cogeneración de electricidad en la fábricas de papel como opción para la reducción del impacto ambiental", III Congreso Brasileiro de Planeamiento Energético, Sao Paulo, Brasil, 1998.
4. Aranda Usón, A., Martínez Gracia, A., Scarpellini, S., Valero Delgado, A., Zabalza Bribián, I., "Disminución de costes energéticos en la Empresa. Tecnología y estrategias para el ahorro y la eficiencia energética", editorial Fundación Confemetal, Madrid 2006.

5. Delgado Acevedo, A. "Perspectiva de la Eficiencia Energética en Calderas en el Sector Pesquero", Conferencia Técnica Mejoras Tecnológicas en la Eficiencia Energética de Calderas Industriales en el Sector Pesquero, Áncash, Perú, 2013.

6. Moreno P. R., Rubio P. R., "Nuevas Tecnologías en Calderas y Eficiencia Energética", Conferencia Técnica Mejoras Tecnológicas en la Eficiencia Energética de Calderas Industriales en el Sector Pesquero, Áncash, Perú, 2013.

7. Telemecanique - Schneider Electric, "Rele programable Zelio-Logic, manual del usuario", editorial Schneider Electric, 2000.

8. Liam Lim, "PET-70216/ET-7026 User Manual, version 1.0.1 beta1", editorial ICP DAS Co, 2010.

9. Indusoft Web Studio v7.1, "User Guide and Technical Reference", editorial Indusoft, 2012.

10. Alba Gonzáles, M., Sandoval Carrillo, A., Martínez Sánchez, D., "Análisis de riesgos del tratamiento del agua para calderas", 1era edición, editorial Plaza y Valdés. México D.F. 1999.

Correspondencia: aldodelgado@peru.com

Recepción de originales: febrero 2014

Aceptación de originales: mayo 2014 